Pacific Journal of Mathematics

A DUALITY FOR QUOTIENT DIVISIBLE ABELIAN GROUPS 


\title{
A DUALITY FOR QUOTIENT DIVISIBLE ABELIAN GROUPS OF FINITE RANK
}

\author{
DAVID M. ARNOLD
}

\begin{abstract}
The usual duality for finite dimensional vector spaces induces a duality $F$ on the category of torsion free quotient divisible abelian groups of finite rank with quasi-homomorphisms as morphisms. This duality preserves rank, is exact, hence preserves quasi-direct sums, sends free groups to divisible groups and conversely, and has the property that for all primes $p, p$-rank $F A=\operatorname{rank} A-p$-rank $A$.
\end{abstract}

A torsion free abelian group is quotient divisible if $A$ has a free subgroup $B$ such that $A / B$ is the direct sum of a torsion divisible group and a group of bounded order. Let $\mathscr{C}$ be the category of quotient divisible abelian groups of finite rank (rank $A$ is the cardinality of a maximal independent subset of $A$ ) with morphism sets $Q \otimes_{z} \operatorname{Hom}(A, B)$, where $Q$ is the field of rational numbers. Morphisms in $\mathscr{C}$ are quasi-homomorphisms of groups.

Theorem A: There is a contravariant exact functor $F: \mathscr{C} \rightarrow \mathscr{C}$ such that $F^{2}$ is naturally equivalent to the identity functor on $\mathscr{C}$, rank $A=\operatorname{rank} F A$ and $A$ is free iff $F A$ is divisible.

Let $R_{p}=\{m / n \in Q \mid(p, n)=1\}$ be the localization of $Z$ at a prime $p$ and $\mathscr{C}_{p}=\left\{A_{p}=R_{p} \otimes_{z} A \mid A \in \mathscr{C}\right\}$ be a category with morphism sets $Q \bigotimes_{R_{p}} \operatorname{Hom}\left(A_{p}, B_{p}\right)$. The duality $F$ induces a duality on $\mathscr{C}_{p}$ which coincides with the duality given in [1].

For $A \in \mathscr{C}, p$-rank $A$ is the $Z / p Z$ dimension of $A / p A$.

Corollary B: For all primes $p, p$-rank $F A=\operatorname{rank} A-p$-rank $A$.

Notation is established in 1 and the relevant results of BeaumontPierce [2] are summarized in a series of lemmas. The proofs of Theorem A and Corollary B are contained in 2. Section 3 includes some easy consequences of the properties of the duality $F$.

1. Preliminaries. The ring of $p$-adic integers, $p$ a prime, is denoted by $R_{p}^{*}$ and $Q_{p}^{*}$ is the quotient field of $R_{p}^{*}$, i.e., the $p$-adic completion of $Q$. There are subring inclusions $Z \subset R_{p} \subset Q \subset Q_{p}^{*}$ and $R_{p} \subset R_{p}^{*} \subset Q_{p}^{*}$ such that $R_{p}^{*} \cap Q=R_{p}, \cap\left\{R_{p} \mid p\right.$ a prime $\}=Z$.

Each finite dimensional $Q$-vector space $V$ may be regarded as a $Q$-subspace of $V_{p}^{*}=Q_{p}^{*} \otimes V$ by identifying $v$ with $1 \otimes v$. If $X$ is a subset of $V$ and $R$ a subring of $Q_{p}^{*}$ then $R X=\left\{\sum r_{i} x_{i} \mid r_{i} \in R, x_{i} \in X\right\}$ 
is an $R$-submodule of $V_{p}^{*}$. Hence $Z X \subset R_{p} X \subset Q X \subset V$ and $R_{p} X \subset R_{p}^{*} X \subset$ $V_{p}^{*}$. Further, if $A$ is a subgroup of $V$ such that $V / A$ is torsion then $R_{p}^{*} V=V_{p}^{*}=Q_{p}^{*} Q A=Q_{p}^{*} A$ and $\operatorname{rank} A=Q$-dimension of $V=Q_{p}^{*}$ dimension of $V_{p}^{*}=R_{p}^{*}$-rank of $R_{p}^{*} A$.

For the remainder of this note, $V$ is a finite dimensional $Q$-vector space, $X$ is a basis of $V$ and $\delta_{p}$ is a $Q_{p}^{*}$-subspace of $V_{p}^{*}$. Define $(X$, $V, \delta)=V \cap\left(\cap\left\{R_{p}^{*} X+\delta_{p} \mid p\right.\right.$ is a prime $\left.\}\right)$.

Lemma 1. Let $A=(X, V, \delta)$ for some $X, V$ and $\delta$.

(a) $R_{p} A=V \cap\left(R_{p}^{*} X+\delta_{p}\right)$;

(b) $R_{p}^{*} A=R_{p}^{*} X+\delta_{p}$ and $\delta_{p}=\cap\left\{p^{i}\left(R_{p}^{*} A\right) \mid i=1,2, \cdots\right\}$;

(c) $A \in \mathscr{C}$ and $Z X$ is a free subgroup of $A$ with $A / Z X$ torsion divisible;

(d) If $Y$ is another basis of $V$ and $B=(Y, V, \delta)$ then there are nonzero integers $m$ and $n$ with $m A \subset B$ and $n B \subset A$.

Proof. Beaumont-Pierce [2], §5.

Lemma 2. Every $A \in \mathscr{C}$ is an $(X, V, \delta)$ for some $X, V$ and $\delta$.

Proof. Choose $V$ such that $A \subset V, V / A$ torsion; let $X$ be a maximal $Z$-independent subset of $A$ with $A / Z X$ torsion divisible and let $\delta_{p}=\cap$ $\left\{p^{i}\left(R_{p}^{*} A\right) \mid i=1,2, \cdots\right\}$. Then $R_{p}^{*} A=R_{p}^{*} X+\delta_{p}$ and $R_{p} A=R_{p}^{*} A \cap V$ for all primes $p$. Hence $A=\cap\left\{R_{p} A \mid p\right.$ prime $\}=\{X, V, \delta\}$.

Note that if $A=(X, V, \delta)$ then $p$-rank $A=\operatorname{rank} A-\left(Q_{p}^{*}\right.$-dimension of $\delta_{n}$ ).

Let $A$ and $B$ be torsion free abelian groups. Call $\phi: A \rightarrow B$ a quasi-homomorphism if there is $0 \neq n \in Z$ with $n \dot{\phi} \in \operatorname{Hom}(A, B)$. Observe that $\{\phi \mid \phi: A \rightarrow B$ is a quasi-homomorphism $\}$ may be identified with $Q \otimes$ Hom $(A, B)$. The groups $A$ and $B$ are quasi-isomorphic $(A \dot{\sim} B)$ if there are monomorphisms $f: A \rightarrow B, g: B \rightarrow A$ such that $B / f(A)$ and $A / g(B)$ are bounded.

Assume that $A=(X, V, \delta)$ and $B=(Y, U, \sigma)$ are objects of $\mathscr{C}$ and that $\phi: A \rightarrow B$ is a quasi-homomorphism. Then $\phi$ induces a unique $Q$-linear transformation $\lambda: V \rightarrow U$ since $V / A$ and $U / B$ are torsion. Define $\phi_{p}=1 \otimes \lambda: V_{p}^{*} \rightarrow U_{p}^{*}$, a $Q_{p}^{*}$-linear transformation extending $\lambda$, hence $\dot{\phi}$. There is an integer $n$ such that $n \dot{\phi}_{p}\left(R_{p}^{*} A\right) \subset R_{p}^{*} B$ so that $\phi_{p}\left(\delta_{p}\right) \subset \sigma_{p}$ for all primes $p$.

Conversely if $\theta: V \rightarrow U$ is a $Q$-linear transformation such that $\theta_{p}\left(\delta_{p}\right) \subset \sigma_{p}$ (where $\theta_{p}=1 \otimes \theta: V_{p}^{*} \rightarrow V_{p}^{*}$ ) for all primes $p$, then $\theta: A \rightarrow$ $B$ is a quasi-homomorphism. Observe that if $W$ is a basis of $U$ with $\theta(X) \subset W$ then $\theta(A) \subset D=(W, U, \sigma)$. By Lemma 1.d, there is $0 \neq$ $n \in Z$ with $n \theta(A) \subset n D \subset B=(Y, U, \sigma)$.

Note that a quasi-homomorphism $\phi: A \rightarrow B$ is a quasi-isomorphism 
iff $\lambda: V \rightarrow U$ is an isomorphism and $\phi_{p}\left(\delta_{p}\right)=\sigma_{p}$ for all primes $p$, where $\lambda$ is the unique extension of $\phi$ and $\phi_{p}=1 \otimes \lambda$.

We summarize some of the categorical properties of $\mathscr{C}$, as given by Walker [4]. Assume that $\phi: A \rightarrow B$ is a quasi-homomorphism and that $f=n \phi \in \operatorname{Hom}(A, B): \phi$ is epic in $\mathscr{C}$ iff $B / f(A)$ is bounded; $\phi$ is monic in $\mathscr{C}$ iff $f$ is monic and $0 \rightarrow A \stackrel{\phi}{\rightarrow} B \stackrel{\theta}{\rightarrow} C \rightarrow 0$ is exact in $\mathscr{C}$ iff $\phi$ is monic, $\theta$ is epic and $(\operatorname{im} f+\operatorname{ker} g) /(\operatorname{im} f) \cap(\operatorname{ker} g)$ is bounded, where $g=m \theta \in \operatorname{Hom}(B, C)$. The direct sum in $\mathscr{C}$ is the quasi-direct sum of groups, $A \oplus \dot{\oplus}$, where $M=A \oplus B$ iff there are non-zero integers $m$ and $n$ with $m M \subset A \oplus B$ and $n(A \oplus B) \subset M$. A group $A \in \mathscr{C}$ is strongly indecomposable if $A$ is indecomposable in $\mathscr{C}$, i.e., $A=B \dot{\oplus} C$ implies that $B=0$ or $C=0$.

Lemma 3. Suppose that $A_{i}=\left(X_{i}, V_{i}, \delta_{i}\right) \in \mathscr{C}, i=1,2,3$. Then $0 \rightarrow A_{1} \stackrel{\phi_{1}}{\rightarrow} A_{2} \stackrel{\phi_{2}}{\longrightarrow} A_{3} \rightarrow 0$ is exact in $\mathscr{C}$ iff $0 \rightarrow V_{1} \stackrel{\lambda_{1}}{\rightarrow} V_{2} \stackrel{\lambda_{2}}{\rightarrow} V_{3} \rightarrow 0$ is an exact sequence of $Q$-vector spaces where $\lambda_{i}$ is the unique extension of $\phi_{i}, i=$ $1,2$.

Proof. Observe that $\phi_{1}$ monic iff $\lambda_{1}$ monic; $\phi_{2}$ epic iff $\lambda_{2}$ epic and $\left(\operatorname{ker} f_{2}+\operatorname{im} f_{1}\right) /\left(\operatorname{ker} f_{2}\right) \cap\left(\operatorname{im} f_{1}\right)$ is bounded iff $\operatorname{ker} \lambda_{2}=\operatorname{im} \lambda_{1}$ where $f_{i}=n_{i} \phi_{i} \in \operatorname{Hom}\left(A_{i}, A_{i+1}\right)$ for $0 \neq n_{i} \in Z, i=1,2$.

2. A Duality for $\mathscr{C}$. Let $\mathscr{V}$ denote the category of finite dimensional $Q$-vector spaces with $Q$-linear transformations as morphisms. Define $G: \mathscr{V} \rightarrow \mathscr{C}$ by $G(V)=V^{\prime}=\operatorname{Hom}_{Q}(V, Q)$; and for $f \in \operatorname{Hom}_{Q}(V$, $U), G(f)=f^{\prime}$ is an element of $\operatorname{Hom}_{Q}\left(U^{\prime}, V^{\prime}\right)$ defined by $f^{\prime}(\alpha)=\alpha f$. It is well-known that $G$ is a contravariant exact functor naturally equivalent to the identity functor on $\mathscr{F}$, i.e., $(f g)^{\prime}=g^{\prime} f^{\prime}$; if $0 \rightarrow U \stackrel{f}{\rightarrow}$ $V \stackrel{g}{\rightarrow} W \rightarrow 0$ is an exact sequence of $Q$-vector spaces then $0 \rightarrow W^{\prime} \stackrel{g^{\prime}}{\rightarrow}$ $V^{\prime} \stackrel{f^{\prime}}{\rightarrow} U^{\prime} \rightarrow 0$ is exact; and for each $V \in \mathscr{Y}$ there is a $Q$-isomorphism $h_{V}: V \rightarrow V^{\prime \prime}$ such that if $f \in \operatorname{Hom}_{2}(V, U), h_{U} f=f^{\prime \prime} h_{V}$. If $\left\{x_{1}, \cdots, x_{n}\right\}$ is a basis for $V$ then $\left\{x_{1}^{\prime}, \cdots, x_{n}^{\prime}\right\}$ is a basis for $V^{\prime}$ where $x_{i}^{\prime}$ is defined by $x_{i}^{\prime}\left(x_{j}\right)=\delta_{i j}$, the Kronecker delta.

Proof of Theorem A.

(a) Definition of $F$. If $A=(X, V, \delta) \in \mathscr{C}$ then there is a $Q_{p}^{*}$ exact sequence

$$
0 \rightarrow \operatorname{Hom}\left(V_{p}^{*} / \delta_{p}, Q_{p}^{*}\right) \stackrel{j_{A}^{\prime}}{\longrightarrow} \operatorname{Hom}\left(V_{p}^{*}, Q_{p}^{*}\right) \stackrel{i_{A}^{\prime}}{\longrightarrow} \operatorname{Hom}\left(\delta_{p}, Q_{p}^{*}\right) \rightarrow 0
$$

induced by the canonical $Q_{p}^{*}$-exact sequence

$$
0 \rightarrow \delta_{p} \stackrel{i_{A}}{\longrightarrow} V_{p}^{*} \stackrel{j_{A}}{\longrightarrow} V_{p}^{*} / \delta_{p} \rightarrow 0 \text {. }
$$


Define $F(A)=\left(X^{\prime}, V^{\prime}, \bar{\delta}\right)$, where $V^{\prime}=\operatorname{Hom}(V, Q), X^{\prime}=\left\{x^{\prime} \mid x \in X\right\}$ and $\bar{\delta}_{p}=j_{A}^{\prime}\left(\operatorname{Hom}\left(V_{p}^{*} / \delta_{p}, Q_{p}^{*}\right)\right)$. Note that $\bar{\delta}_{p}$ may be regarded as a subspace of $\left(V^{\prime}\right)_{p}^{*}$ since Hom $\left(V_{p}^{*}, Q_{p}^{*}\right)$ is naturally isomorphic to $Q_{p}^{*} \otimes V^{\prime}=\left(V^{\prime}\right)_{p}^{*}$.

(b) $F$ is a contravariant functor. Let $B=(Y, U, \sigma), \theta: A \rightarrow B$ a quasi-homomorphism, $\lambda: V \rightarrow U$ the unique extension of $\theta$ and $\theta_{p}=$ $1 \otimes \lambda: V_{p}^{*} \rightarrow U_{p}^{*}$. Define $F(\theta)=\lambda^{\prime} \in \operatorname{Hom}_{Q}\left(U^{\prime}, V^{\prime}\right)$. Then $F(\theta): F(B) \rightarrow$ $F(A)$ is a quasi-homomorphism if for all primes $p, F(\theta)_{p}\left(\bar{\sigma}_{p}\right) \subset \bar{\delta}_{p}$, where $F(\theta)_{p}=1 \otimes \lambda^{\prime}:\left(U^{\prime}\right)_{p}^{*} \rightarrow\left(V^{\prime}\right)_{p}^{*}$.

Since $\theta_{p}\left(\delta_{p}\right) \subset \sigma_{p}$ there is a canonical homomorphism $\phi_{p}: V_{p}^{*} / \delta_{p} \rightarrow$ $U_{p}^{*} / \sigma_{p}$ such that $\phi_{p} j_{A}=j_{B} \theta_{p}$. Thus $j_{A}^{\prime} \phi_{p}^{\prime}=\theta_{p}^{\prime} j_{B}^{\prime}$. It now follows that $F(\theta)_{p}\left(\bar{\sigma}_{p}\right) \subset \bar{\delta}_{p}$ since $\theta_{p}^{\prime}=(1 \otimes \lambda)^{\prime}$ is identified with $1 \otimes \lambda^{\prime}=F(\theta)_{p}$ by the natural isomorphism of (a).

It is now clear that $F$ is a contravariant functor in $\mathscr{C}$, since $G$ is a contravariant functor in $U$.

(c) $F^{2}$ is naturally equivalent to the identity. For $A=(X, V$, $\delta) \in \mathscr{C}$, define $g_{A}: A \rightarrow F^{2} A=\left(X^{\prime \prime}, V^{\prime \prime}, \overline{\bar{\delta}}\right)$ to be the restriction of the $Q$-isomorphism $h_{V}: V \rightarrow V^{\prime \prime}$. It follows that $g_{A}$ is a quasi-isomorphism since $\left(g_{A}\right)_{p}=1 \otimes h_{V}: V_{p}^{*} \rightarrow\left(V^{\prime \prime}\right)_{p}^{*}$ has the property that $\left(g_{A}\right)_{p}\left(\delta_{p}\right)=\overline{\overline{\delta_{p}}}$.

Let $\theta: A \rightarrow B=(X, U, \sigma)$ be a quasi-homomorphism. Then $g_{B} \theta=$ $F^{2}(\theta) g_{A}$ since $h_{U} \lambda=\lambda^{\prime \prime} h_{V}$, where $\lambda$ is the unique extension of $\theta, \lambda: V \rightarrow U$. Therefore, $F^{2}$ is naturally equivalent to the identity functor on $\mathscr{C}$.

(d) $F$ is exact. Assume $0 \rightarrow A_{1} \stackrel{\phi_{1}}{\longrightarrow} A_{2} \stackrel{\phi_{2}}{\longrightarrow} A_{3} \rightarrow 0$ is an exact sequence in $\mathscr{C}$. By Lemma $3,0 \rightarrow V_{1} \stackrel{\lambda_{1}}{\rightarrow} V_{2} \stackrel{\lambda_{2}}{\rightarrow} V_{3} \rightarrow 0$ is exact hence $0 \rightarrow V_{3}^{\prime} \stackrel{\lambda_{2}^{\prime}}{\rightarrow}$ $V_{2}^{\prime} \stackrel{\lambda_{1}^{\prime}}{\rightarrow} V_{1}^{\prime} \rightarrow 0$ is exact. Again by Lemma $3,0 \rightarrow F\left(A_{3}\right) \stackrel{F\left(\phi_{2}\right)}{\longrightarrow} F\left(A_{2}\right) \stackrel{F\left(\phi_{1}\right)}{\longrightarrow}$ $F\left(A_{1}\right) \rightarrow 0$ is exact. Consequently, $F$ is an exact functor.

(e) $A$ is free iff $F A$ is divisible. Observe that $A=(X, V, \delta)$ is free iff $\delta_{p}=0$ for all primes $p$ and divisible iff $\delta_{p}=R_{p}^{*} A$ for all primes $p$.

Proof of Corollary B. A consequence of the definition of $F$ and Lemma 3.

Note that $A$ is strongly indecomposable iff $F A$ is strongly indecomposable.

3. Examples and applications. If $A$ is a rank 1 quotient divisible group with type $\left(k_{i}\right)$, then $k_{i}=0$ or $\infty$. It is easy to see that $F A$ is a rank 1 quotient divisible group with type $\left(l_{i}\right)$ where $l_{i}=0$ if $k_{i}=\infty$ and $l_{i}=\infty$ if $k_{i}=0$. 
A torsion free abelian group $A$ is locally free if $R_{p} A$ is a free $R_{p}$-module for all primes $p$. The only locally free quotient divisible modules of finite rank are free, since if $A$ is such a group $F A$ is divisible $\left(R_{p} F A\right.$ is divisible for all primes $\left.p\right)$ hence $A$ is free.

For $A \in \mathscr{C}$, let $E(A)$ be the quasi-endomorphism ring of $A$. Then $F$ induces a ring anti-isomorphism from $E(A)$ to $E(F A)$ which is an isomorphism if $E(A)$ is commutative.

Beaumont-Pierce [3], Corollary 4.6, prove that a torsion free group $A$, of finite rank, is isomorphic to the additive group of a full subring of a semi-simple rational algebra (i.e., has semi-simple algebra type) iff $A$ is quotient divisible and $A \dot{\sim} B_{1} \oplus \cdots \oplus B_{n}, B_{i}$ strongly indecomposable, and each $E\left(B_{i}\right)$ is an algebraic number field, whose dimension over $Q$ is the rank of $B_{i}$. It follows that $A$ has semi-simple algebra type iff $F A$ does.

One can show, as in [1], that if rank $A=n+1$ and $p$-rank $A=$ $n$ for all primes $p, F(A)=\Lambda^{n} A$, the $n$th exterior power of $A$. A module theoretic characterization of $F$, in general, is unknown to the author.

\section{REFERENCES}

1. D. Arnold, A duality for torsion free modules of finite rank over a discrete valuation Ring, Proc. London Math. Soc., (3), 24 (1972), 204-216.

2. R. A. Beaumont and R. S. Pierce, Torsion Free Rings, Illinois J. Math., 5 (1961), 61-98.

3. - Subrings of algebraic number fields, Acta Sci. Math. Szeged, 22 (1961), 202-216.

4. E. A. Walker, Quotient categories and quasi-isomorphisms of Abelian groups, Proc. of Colloq. on Abelian Groups, Budapest, (1964), 147-162.

Received March 19, 1971 and in revised form August 31, 1971.

New Mexico State University 



\section{PACIFIC JOURNAL OF MATHEMATICS}

\section{EDITORS}

H. SAMELSON

Stanford University

Stanford, California 94305

C. R. HobBY

University of Washington

Seattle, Washington 98105
J. DUGUNDJI

Department of Mathematics

University of Southern California

Los Angeles, California 90007

RICHARD ARENS

University of California

Los Angeles, California 90024

\section{ASSOCIATE EDITORS}

E. F. BECKENBACH

B. H. NeumanN

F WoLF

K. YoshidA

\section{SUPPORTING INSTITUTIONS}

UNIVERSITY OF BRITISH COLUMBIA

UNIVERSITY OF SOUTHERN CALIFORNIA

CALIFORNIA INSTITUTE OF TECHNOLOGY

UNIVERSITY OF CALIFORNIA

MONTANA STATE UNIVERSITY

STANFORD UNIVERSITY

UNIVERSITY OF NEVADA

NEW MEXICO STATE UNIVERSITY

OREGON STATE UNIVERSITY

UNIVERSITY OF OREGON

OSAKA UNIVERSITY

UNIVERSITY OF TOKYO

UNIVERSITY OF UTAH

WASHINGTON STATE UNIVERSITY

UNIVERSITY OF WASHINGTON

$\stackrel{*}{*} \stackrel{*}{*} \stackrel{*}{*}{ }^{*}{ }^{*}$ MMEICAN MATHEMATICAL SOCIETY

NAVAL WEAPONS CENTER

Printed in Japan by International Academic Printing Co., Ltd., Tokyo, Japan 


\section{Pacific Journal of Mathematics}

\section{Vol. 42, No. $1 \quad$ January, 1972}

Tage Bai Andersen, On Banach space valued extensions from split faces ........

David Marion Arnold, A duality for quotient divisible abelian groups of finite

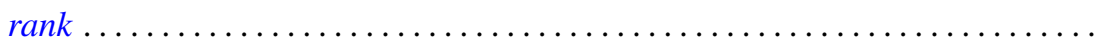

Donald Pollard Ballou, Shock sets for first order nonlinear hyperbolic

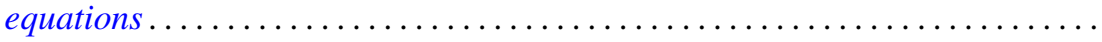

Leon Brown and Lowell J. Hansen, On the range sets of $H^{p}$ functions .........

Alexander Munro Davie and Arne Stray, Interpolation sets for analytic

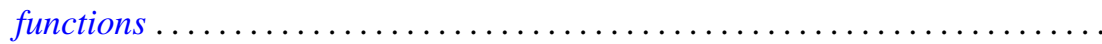

M. G. Deshpande, Structure of right subdirectly irreducible rings. II . . . . . . . . .

Barry J. Gardner, Some closure properties for torsion classes of abelian



Paul Daniel Hill, Primary groups whose subgroups of smaller cardinality are

direct sums of cyclic groups . . . . . . . . . . . . . . . . . . .

Richard Allan Holzsager, When certain natural maps are equivalences .........



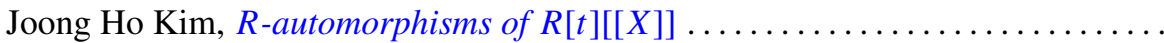

Shin'ichi Kinoshita, On elementary ideals of polyhedra in the 3-sphere.........

Andrew T. Kitchen, Watts cohomology and separability...

Vadim Komkov, A technique for the detection of oscillation of second order

ordinary differential equations .

Charles Philip Lanski and Susan Montgomery, Lie structure of prime rings of characteristic 2

Andrew Lenard, Some remarks on large Toeplitz determinants . .

Kathleen B. Levitz, A characterization of general Z.P.I.-rings. II .

Donald A. Lutz, On the reduction of rank of linear differential systems

David G. Mead, Determinantal ideals, identities, and the Wronskian ...

Arunava Mukherjea, A remark on Tonelli's theorem on integration in product



Hyo Chul Myung, A generalization of the prime radical in nonassociative rings.

John Piepenbrink, Rellich densities and an application to unconditionally nonoscillatory elliptic equations.

Michael J. Powers, Lefschetz fixed point theorems for a new class of multi-valued maps .

Aribindi Satyanarayan Rao, On the absolute matrix summability of a Fourier



T. S. Ravisankar, On Malcev algebras ......................... 227

William Henry Ruckle, Topologies on sequences spaces . . . . . . . . . . . . . 235

Robert C. Shock, Polynomial rings over finite dimensional rings . . . . . . . . . 251

Richard Tangeman, Strong heredity in radical classes . . . . . . . . . . . . . . 259

B. R. Wenner, Finite-dimensional properties of infinite-dimensional spaces . . . . 267 